\title{
Diagnostic value and imaging features of multi-detector CT in lung adenocarcinoma with ground glass nodule patients
}

\author{
JUN LU $^{1 *}$, HAITAO TANG ${ }^{2 *}$, XINGUO YANG ${ }^{1}$, LEI LIU $^{1}$ and MINXIA PANG ${ }^{1}$ \\ Departments of ${ }^{1}$ Radiology and ${ }^{2}$ Surgery, Shengli Oilfield Central Hospital, Dongying, Shandong 257034, P.R. China
}

Received June 14, 2019; Accepted April 8, 2020

DOI: $10.3892 / 01.2020 .11631$

\begin{abstract}
This study investigated the application value and imaging features of multi-detector CT (MDCT) in the treatment of lung adenocarcinoma with ground glass nodules (GGN). The medical data of 168 patients with pulmonary GGN in Shengli Oilfield Central Hospital from January 2013 to June 2015 were analyzed. Patients with microinvasive adenocarcinoma and invasive adenocarcinoma were included in group A (invasive lung adenocarcinoma, $\mathrm{n}=98$ ), while patients with atypical adenomatous hyperplasia and adenocarcinoma in situ were included in group B (pre-invasive lung adenocarcinoma, $\mathrm{n}=70$ ). The imaging features of MDCT were compared. ROC curves of the size of nidus and the size of solid component were drawn for the diagnosis of invasive lung adenocarcinoma. Logistic multivariate regression analysis was used to analyze the risk factors that affected invasive lung adenocarcinoma. There were significant differences in nidus, burr, and lobes of the patients between groups A and B. The size of nidus and the size of solid component of the patients in group A were significantly higher than those of the patients in group B. The AUCs of the size of the nidus and the size of the solid component of the invasive lung adenocarcinoma were 0.891 and 0.902 , respectively. The AUC of the combined diagnosis was 0.984 . Size of the nidus, size of the solid component, nature of the lesion, burr, and lobes were all risk factors for invasive lung adenocarcinoma. In patients with GGN, size of the nidus and size of the solid component can be used as excellent diagnostic parameters for invasive lung adenocarcinoma, and nidus size ( $\geq 9.8 \mathrm{~mm}$ ), size of the solid component $(\geq 0.9 \mathrm{~mm})$, the mixed GGN nature of the nidus, burr and lobes can distinguish invasive lung adenocarcinoma and pre-invasive lesions.
\end{abstract}

Correspondence to: Dr Minxia Pang, Department of Radiology, Shengli Oilfield Central Hospital, 31 Jinan Road, Dongying, Shandong 257034, P.R. China

E-mail: tgkw74@163.com

*Contributed equally

Key words: ground glass nodule, pre-invasive lung adenocarcinoma, invasive lung adenocarcinoma, multi-detector CT, diagnostic value, imaging feature

\section{Introduction}

Lung cancer consists of non-small cell lung cancer and small cell lung cancer. Non-small cell lung cancer accounts for about $85 \%$ in lung cancer $(1,2)$. The subtype of non-small cell lung cancer is generally lung adenocarcinoma, which accounts for about $40 \%$ in lung cancer (3). At present, the early diagnosis of patients with lung adenocarcinoma is still poor. Many patients are in the advanced stage or have metastasis when they are diagnosed, which makes the treatment of patients very difficult $(4,5)$. At present, small pulmonary nodules are treated by non-invasive methods such as chest computed tomography (CT) and MRI and invasive diagnostic methods such as biopsy and puncture (6). Chest CT is an optimal method to screen lung cancer, and has high resolution. The detection rate of lung ground glass nodules (GGN) is increasing with the application of chest CT (7).

GGN lesion can be divided into pure GGN (pGGN) lesion and mixed GGN (mGGN) lesion in CT imaging. It is shown that the malignant rate of GGN of early lung adenocarcinoma is high in thin slice CT. A total of $18 \%$ of pGGN and $63 \%$ of mGGN are malignant niduses (8). According to the classification criteria of International Association for the Study of Lung Cancer (IASLC), American Thoracic Society (ATS), and European Respiratory Society (ERS), lung adenocarcinoma can be divided into atypical adenomatous hyperplasia (AAH), adenocarcinoma in situ (AIS), microinvasive adenocarcinoma (MIA), and invasive adenocarcinoma (IAC) $(9,10)$. AAH and AIS are considered as pre-invasive lung adenocarcinoma, and MIA and IAC as invasive lung adenocarcinoma. However, there are pGGN or mGGN in AAH, AIS, MIA, and IAC and there are different solid components in CT imaging $(11,12)$. There are significant differences between the operation of pre-invasive lung adenocarcinoma and the operation of invasive lung adenocarcinoma. Also, the lymph node structure of patients with pre-invasive lung adenocarcinoma or invasive lung adenocarcinoma is different $(13,14)$. Therefore, it is crucial to discriminate invasive lesions and invasive lung adenocarcinoma accurately, especially in terms of operation plan, prognosis assessment, and doctor-patient communication.

With the development of CT technology, multi-detector CT (MDCT) is used to screen tumors, because its time resolution is good, and it can process data in a same direction and has various image processing functions (15). In the present study, MDCT was used to examine GGN patients. The imaging features of MDCT and its predictive value for pre-invasive lung adenocarcinoma and invasive lung adenocarcinoma were investigated. 
Table I. General data of the patients in groups A and B, $\mathrm{n}(\%) /($ mean $\pm \mathrm{SD})$.

\begin{tabular}{|c|c|c|c|c|}
\hline Categories & Group A $(\mathrm{n}=98)$ & Group B $(n=70)$ & $t / \chi^{2}$ & P-value \\
\hline Sex & & & 0.250 & 0.617 \\
\hline Male & $41(41.84)$ & $32(45.71)$ & & \\
\hline Female & $57(58.16)$ & $38(54.29)$ & & \\
\hline Age (years) & $57.3 \pm 6.2$ & $57.9 \pm 6.1$ & 0.534 & 0.623 \\
\hline BMI $\left(\mathrm{kg} / \mathrm{m}^{2}\right)$ & $22.26 \pm 3.59$ & $22.89 \pm 3.47$ & 1.137 & 0.257 \\
\hline Smoking history & & & 0.428 & 0.513 \\
\hline Yes & $47(47.96)$ & $30(42.86)$ & & \\
\hline No & $51(52.04)$ & $40(57.14)$ & & \\
\hline Respiratory symptoms & & & 0.744 & 0.388 \\
\hline Yes & $19(19.39)$ & $10(14.29)$ & & \\
\hline No & $79(80.61)$ & $60(85.71)$ & & \\
\hline Family history of cancers & & & 0.520 & 0.471 \\
\hline Yes & $15(15.31)$ & $8(11.43)$ & & \\
\hline No & $83(84.69)$ & $62(88.57)$ & & \\
\hline $\mathrm{CEA}(\mathrm{ng} / \mathrm{ml})$ & $3.16 \pm 1.54$ & $2.84 \pm 1.35$ & 1.397 & 0.164 \\
\hline $\operatorname{AST}(\mathrm{U} / \mathrm{l})$ & $21.07 \pm 11.27$ & $20.25 \pm 10.56$ & 0.477 & 0.634 \\
\hline $\operatorname{ALT}(\mathrm{U} / \mathrm{l})$ & $27.63 \pm 9.48$ & $28.41 \pm 9.24$ & 0.531 & 0.596 \\
\hline
\end{tabular}

BMI, body mass index; SD, standard deviation; ALT, alanine aminotransferase; AST, aspartate aminotransferase; CEA, carcinoembryonic antigen.

\section{Patients and methods}

General data. The data of 168 patients with pulmonary GGN were analyzed. These patients were diagnosed in Shengli Oilfield Central Hospital (Dongying, China) from January 2013 to June 2015. According to the classification criteria of IASLC/ATS/ERS (16), there were 11 patients with AAH, 59 patients with AIS, 29 patients with MIA, and 69 patients with IAC. Patients with MIA and IAC were included in group A (invasive lung adenocarcinoma, $n=98$ ), and patients with AAH and AIS were included in group B (pre-invasive lung adenocarcinoma, $\mathrm{n}=70$ ). There were 38 patients with pGGN and 60 patients with mGGN in group A, including 41 males and 57 females, aged 36 to 81 years, with a mean age of $(57.3 \pm 6.2)$. There were 51 patients with pGGN and 19 patients with mGGN in group B, including 32 males and 38 females, aged 35 to 79 years, with a mean age of $(57.9 \pm 6.1)$.

This study was approved by the Ethics Committee of Shengli Oilfield Central Hospital. Signed informed consent was obtained from the patients or their guardians.

Inclusion and exclusion criteria. Inclusion criteria: Patients with lung adenocarcinoma according to postoperative histopathological diagnosis (17); with complete medical record; without primary tumors and patients with blood sugar levels at normal levels. Exclusion criteria: Patients with history of iodine allergy and other drug allergies; with history of thoracic surgery; with other tumors; complicated with severe liver dysfunction and kidney dysfunction; with acute pulmonary infection, connective tissue diseases, infectious diseases, metabolic diseases, hematopoietic dysfunction and patients with mental illness or a family history of mental illness, pregnant or lactating women.

MDCT examination. Patients were scanned by a SOMATOM Definition AS+ 64-row 128-slice spiral CT scanner (Siemens Healthcare $\mathrm{GmbH}$ ). The patients took a supine position, with their heads close to the scanner, then they took a deep breath and held the breath. Scope: Between the sternoclavicular joint and the base of lungs, including the chest wall and armpits on both sides. The sternoclavicular joint was used as a baseline. Scanning parameters: Tube current $170 \mathrm{mAs}$; tube voltage $120 \mathrm{kV}$; visual field $350 \mathrm{~mm}$; layer thickness $5 \mathrm{~mm}$; and reconstruction thickness $2 \mathrm{~mm}$. Subsequently, an enhanced examination was performed. The contrast agent, iopamidol with a concentration of $1.5 \mathrm{ml} / \mathrm{kg}$, was injected into the elbow vein of the patients by a high pressure injector at a rate of $2.5 \mathrm{ml} /$ $\mathrm{sec}$, and the dosage was between 80 and $100 \mathrm{ml}$ (Shanghai Bracco Sine Pharmaceutical Corp. Ltd.; H20053388). After this step was finished, the patients took a deep breath and held the breath, then their lungs were scanned. The window level of mediastinal imaging was $220 \mathrm{HU}$, the window width was $40 \mathrm{HU}$. The width of the lung window was $1,600 \mathrm{HU}$, the window level was $-600 \mathrm{HU}$. After the patients were scanned, the data were input into Extended Brilliance ${ }^{\mathrm{TM}}$ workstation and the imaging was rebuilt.

Imaging analysis. Data were observed, including type of nidus (single and multiple), site of nidus (left, right), size of nidus (the length, diameter, axis of nidus and the length of solid component), size of solid component (the diameter of solid component), nature of nidus (pGGN, mGGN), pleura, burr, 
Table II. Comparison of the imaging features of MDCT of the patients in groups A and B (mean \pm SD).

\begin{tabular}{|c|c|c|c|c|}
\hline Categories & Group A $(\mathrm{n}=98)$ & Group B $(n=70)$ & $t / \chi^{2}$ & P-value \\
\hline Type of nidus & & & 0.049 & 0.826 \\
\hline Single & 77 (78.57) & $54(77.14)$ & & \\
\hline Multiple & $21(21.43)$ & $16(22.86)$ & & \\
\hline Site of nidus & & & 0.334 & 0.563 \\
\hline Right & $53(54.08)$ & $41(58.57)$ & & \\
\hline Left & $45(45.92)$ & $29(41.43)$ & & \\
\hline Size of nidus (mm) & $12.3 \pm 2.4$ & $8.6 \pm 3.9$ & 7.596 & $<0.001$ \\
\hline Size of solid component (mm) & $1.6 \pm 0.8$ & $0.6 \pm 0.4$ & 9.628 & $<0.001$ \\
\hline Nature of nidus & & & 19.040 & $<0.001$ \\
\hline pGGN & 38 (38.78) & $51(72.86)$ & & \\
\hline mGGN & $60(61.22)$ & $19(27.14)$ & & \\
\hline Pleural indentation & & & 3.323 & 0.068 \\
\hline Yes & $10(10.20)$ & $2(2.86)$ & & \\
\hline No & $88(89.80)$ & $68(97.14)$ & & \\
\hline Burr & & & 21.661 & $<0.001$ \\
\hline Yes & $36(36.73)$ & $4(5.71)$ & & \\
\hline No & $62(63.27)$ & $66(94.29)$ & & \\
\hline Lobes & & & 23.851 & $<0.001$ \\
\hline Yes & $72(73.47)$ & $25(35.71)$ & & \\
\hline No & $26(26.53)$ & $45(64.29)$ & & \\
\hline Clear periphery & & & 2.661 & 0.103 \\
\hline Yes & $81(82.65)$ & $64(91.43)$ & & \\
\hline No & $17(17.35)$ & $6(8.57)$ & & \\
\hline Vacuole & & & 3.680 & 0.055 \\
\hline Yes & $36(36.73)$ & $16(22.86)$ & & \\
\hline No & $62(63.27)$ & $54(77.14)$ & & \\
\hline Similar round & & & 1.721 & 0.190 \\
\hline Yes & $65(66.33)$ & $53(75.71)$ & & \\
\hline No & $33(33.67)$ & $17(24.29)$ & & \\
\hline
\end{tabular}

MDCT, multi-detector CT; SD, standart deviation; mGGN, mixed ground glass nodules; pGGN, pure ground glass nodules.

lobe, periphery, vacuole, and similar round. CT imaging was analyzed by two radiologists.

Statistical analysis. Statistical analysis was performed by SPSS 22.0 (IBM Corp). The count data were expressed as [n (\%)]. Chi-square test was used to compare the count data between groups. The measurement data were expressed as mean value \pm standard deviation (mean \pm SD). Independent sample t-test was used to compare the measurement data between groups. ROC curves of the size of nidus and size of solid component were drawn. Logistic multivariate regression analysis was used to analyze the risk factors that affect invasive lung adenocarcinoma. $\mathrm{P}<0.05$ indicates that the difference is statistically significant.

\section{Results}

General data of the patients in two groups. There was no significant difference in general data of the patients in groups
A and B, including sex, age, body mass index, smoking history, respiratory symptoms, family history of cancers, carcinoembryonic antigen (CEA), aspartate aminotransferase (AST) and alanine aminotransferase (ALT) $(\mathrm{P}>0.05)$ (Table I).

Imaging features of MDCT of the patients in two groups. Size of nidus of patients in group B was smaller, many patients in group B had pGGN. Many patients in group A had mGGN. There were no or only a few solid components in mGGN in the plain scan. Solid components could be seen after CT was enhanced. In the imaging of MDCT of patients in group A and $\mathrm{B}$, there was no significant difference in the type of nidus, site of nidus, pleural indentation, clear periphery, vacuole, and the ratio of similar round $(\mathrm{P}>0.05)$. There were significant differences in the nature of nidus, burr, and lobes of patients in group A and B $(\mathrm{P}<0.05)$. Sizes of nidus and solid components of patients in group A were significantly bigger than those of patients in group $\mathrm{B}(\mathrm{P}<0.05)$ (Table II). 

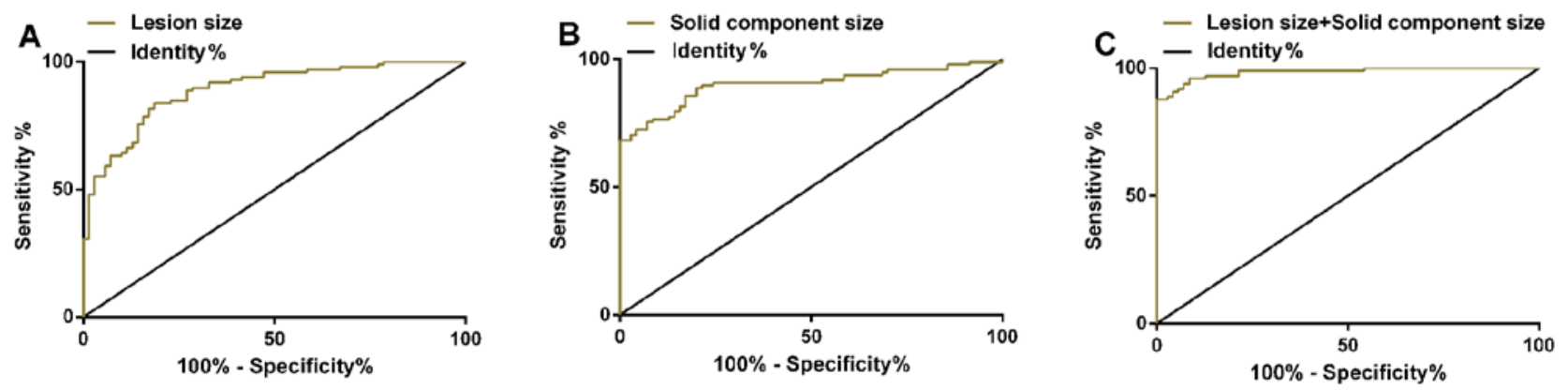

Figure 1. ROC curves of the size of nidus and the size of solid component in the diagnosis of invasive lung adenocarcinoma. (A) Sensitivity of the size of nidus was $83.67 \%$ and specificity was $81.43 \%$ in the diagnosis of invasive lung adenocarcinoma. (B) Sensitivity of the size of solid component was $88.78 \%$ and specificity was $80.00 \%$ in the diagnosis of invasive lung adenocarcinoma. (C) Sensitivity and specificity of the nidus combined with the size of the solid component for the diagnosis of invasive lung adenocarcinoma were 87.76 and $100.00 \%$, respectively.

Table III. The diagnostic value of the size of nidus and the size of solid component for invasive lung adenocarcinoma.

\begin{tabular}{lcccccc}
\hline Parameters & AUC & $95 \%$ CI & SE & Cut-off & Sensitivity (\%) & Specificity (\%) \\
\hline Size of nidus & 0.891 & $0.843-0.939$ & 0.024 & $9.8(\mathrm{~mm})$ & 83.67 & 81.43 \\
$\begin{array}{l}\text { Size of solid component } \\
\text { Size of nidus + size }\end{array}$ & 0.902 & $0.854-0.949$ & 0.024 & $0.9(\mathrm{~mm})$ & 88.78 & 80.00 \\
of solid component & & $0.969-0.999$ & 0.001 & 0.9 & 87.76 & 100.00 \\
\hline
\end{tabular}

AUC, area under the ROC curve; CI, confidence intervals; SE, standard error.

Table IV. Logistic multivariate regression analysis and assignments.

\begin{tabular}{lcc}
\hline Factors & Variate & Assignments \\
\hline Size of nidus (mm) & X1 & $\geq 9.8=1,<9.8=0$ \\
Size of solid component (mm) & X2 & $\geq 0.9=1,<0.9=0$ \\
Nature of nidus & X3 & mGGN $=1, \mathrm{pGGN}=0$ \\
Burr & X4 & Yes $=1$, No $=0$ \\
Lobes & X5 & Yes $=1$, No=0 \\
\hline
\end{tabular}

mGGN, mixed ground glass nodules; pGGN, pure ground glass nodules.

Diagnostic value of the size of nidus and the size of solid component for invasive lung adenocarcinoma. ROC curves of the size of nidus and the size of solid component in invasive lung adenocarcinoma were drawn. AUC of the size of nidus in the treatment of invasive lung adenocarcinoma was 0.891 (95\% CI: $0.843-0.939$ ), and sensitivity was $83.67 \%$; specificity was $81.43 \%$, and optimal cut-off value was $9.8 \mathrm{~mm}$. AUC of the size of solid component in the treatment of invasive lung adenocarcinoma was 0.902 (95\% CI: 0.854-0.949), and sensitivity was $88.78 \%$; specificity was $80.00 \%$, and the optimal cut-off value was $0.9 \mathrm{~mm}$. ROC curve was plotted to investigate the diagnosis of the size of nidus combined with the size of the solid component for invasive lung adenocarcinoma. The combined AUC was 0.984 (95\% CI: 0.969-0.999); sensitivity was $87.76 \%$; and specificity was $100.00 \%$ (Table III and Fig. 1).
Logistic multivariate regression analysis of risk factors of invasive lung adenocarcinoma. Logistic multivariate regression analysis was used to analyze different factors in pre-invasive lung adenocarcinoma and invasive lung adenocarcinoma. The result showed that the size of nidus (OR: $2.834,95 \% \mathrm{CI}$ : $1.577-5.092)$, the size of solid component (OR: $16.605,95 \%$ CI: 4.037-20.611), the nature of nidus (OR: $2.998,95 \%$ CI: $1.734-$ 5.183), burr (OR: 12.033, 95\% CI: 4.119-17.857), lobes (OR: 3.575, 95\% CI: 1.836-6.085) were risk factors that affected invasive lung adenocarcinoma $(\mathrm{P}<0.05)$ (Tables IV and $\mathrm{V})$.

\section{Discussion}

At present, when CT is used to scan the lungs of patients, its imaging features are deficient for the diagnosis of GGN. AAH, AIS, MIA, and IAC can be manifested as pGGN or mGGN with a small amount of solid components in $\mathrm{CT}$ imaging. It is difficult to diagnose the nature of nidus accurately before operation only according to the imaging features of niduses in CT imaging $(18,19)$.

Histological biopsy is an important method to diagnose whether GGN that existed in the patients' body for a long time is an IAC, but biopsy and histological examination may cause some trauma (20). The operation plans of pre-invasive lesion of lung adenocarcinoma and invasive lung adenocarcinoma are different. Diagnosing and timely treatment of pre-invasive lesion of lung adenocarcinoma and invasive lung adenocarcinoma can avoid unnecessary lymph node dissection and reduce the trauma of patients. Thus, the early cure rate can reach up to $100 \%$ (21). AAH, AIS, and MIA grow along respiratory bronchiolar wall and alveolar wall, and most of them 
Table V. Logistic multivariate regression analysis of risk factors of invasive lung adenocarcinoma.

\begin{tabular}{lccrrc}
\hline Factors & $\beta$ & SE & Wals & P-value & OR (95\% CI) \\
\hline Size of nidus (mm) & 1.042 & 0.299 & 12.128 & $<0.001$ & $2.834(1.577-5.092)$ \\
Size of solid component (mm) & 4.036 & 1.347 & 8.975 & 0.003 & $16.605(4.037-20.611)$ \\
Nature of nidus & 1.098 & 0.279 & 15.457 & $<0.001$ & $2.998(1.734-5.183)$ \\
Burr & 4.344 & 1.426 & 9.284 & 0.002 & $12.033(4.119-17.857)$ \\
Lobes & 2.808 & 0.816 & 11.831 & 0.001 & $3.575(1.836-6.085)$ \\
\hline
\end{tabular}

SE, standard error; CI, confidence intervals.

are pGGN. If there are obvious local fibroblast proliferation, local accumulation of tumor cells or alveolar wall collapse, AAH, AIS, and MIA are often manifested as mGGN $(22,23)$. In this study, there were significant differences between the nature of nidus, burr, and lobes of pre-invasive lung adenocarcinoma and those of invasive lung adenocarcinoma. Pre-invasive lung adenocarcinoma manifests as pGGN, while invasive lung adenocarcinoma manifests as mGGN. The morphological features of non-lobulated periphery and nonburr periphery of some pre-invasive GGN lesions were similar to those in previous studies $(24,25)$. In pulmonary nodules, it is known that large nodules are more malignant than small nodules, and that the solid component of GGN represents a region of fibroblast proliferation or an invasive component of tumors $(26,27)$. In this study, the size of nidus and the size of solid component of patients in group A were significantly larger than those of patients in group B. This conclusion is similar to the conclusion in the study of Yoon et al (28). The sizes of nodules and solid components of MIA and IAC are larger than those of pre-invasive lung adenocarcinoma. ROC curves of nidus size and solid component size for the diagnosis of invasive lung adenocarcinoma were plotted, and it was found that the AUCs of the size of nidus, the size of solid component and the combination for the diagnosis of invasive lung adenocarcinoma were 0.891, 0.902 and 0.984, respectively. It suggested that the size of nidus and the size of solid component can be used as diagnostic parameters of invasive lung adenocarcinoma, and the combination of the two has better diagnostic efficiency. Logistic multivariate regression analysis was used to analyze risk factors of invasive lung adenocarcinoma. The result showed that the size of nidus, the size of solid component, the nature of nidus, burr, and lobes were risk factors of invasive lung adenocarcinoma. In the study of Lee et al (29), it was shown that the nidus with size of $<10 \mathrm{~mm}$ could distinguish pre-invasive lesions and invasive lesions; the smaller nidus size, the smaller solid proportion, the borderline without fissure and the borderline without spots were the significant distinguishing factors of the preinfiltrating lesion. In this study, it was found that the optimal cut-off values for lesion size and solid component size for invasive lung adenocarcinoma were $9.8 \mathrm{~mm}$ and $0.9 \mathrm{~mm}$, respectively. Therefore, we consider that $<10$ and $1 \mathrm{~mm}$ may be helpful in the diagnosis of invasive lung adenocarcinoma. Whether 10 and $1 \mathrm{~mm}$ can be used as alternatives need to be studied in the future. It was found in this study that nidus size combined with solid component size could improve the diagnostic efficacy of invasive lung adenocarcinoma, and confirmed that burr and lobes were risk factors for IAC, which were not reported before. Hu et al (30) used CT and positron emission tomography (PET) to show that the appearance of vacuole signs, pleural indentation signs, lobes, and burr-like appearance indicates that the lesion is more likely to be malignant than benign. For smaller GGN, the detection rate of PET-CT is lower, while for larger GGN the detection rate is higher. This study mainly used MDCT to distinguish between MIA, IAC and AAH, in AIS patients, and did not observe the CT signs of group A and B, which is one of the limitations of this study.

This study confirmed the diagnostic value of MDCT in the treatment of pre-invasive lung adenocarcinoma and invasive lung adenocarcinoma. However, the imaging features of MDCT of patients with AAH, AIS, MIA and IAC were not observed. There are some overlaps in the imaging features of MDCT of pre-invasive lung adenocarcinoma and invasive lung adenocarcinoma. Therefore, in clinical practice, clinical symptoms, signs, cytology, and histological examination should be jointly used to reduce misdiagnosis rate.

In conclusion, in GGN patients, nidus size and solid component size can be used as excellent diagnostic parameters for invasive lung adenocarcinoma, while nidus size $(\geq 9.8 \mathrm{~mm})$, solid component size ( $\geq 0.9 \mathrm{~mm}$ ), mGGN nature of lesion, burr and lobes can distinguish invasive lung adenocarcinoma and preinvasive lesions.

\section{Acknowledgements}

Not applicable.

\section{Funding}

No funding was received.

\section{Availability of data and materials}

The datasets used and/or analyzed during the present study are available from the corresponding author on reasonable request.

\section{Authors' contributions}

JL and HT conceived and designed the study, and drafted the manuscript. JL, XY, LL and MP collected, analyzed and interpreted the experimental data. MP and HT contributed to 
the imaging analysis and revised the manuscript for important intellectual content. All authors read and approved the final version of the manuscript.

\section{Ethics approval and consent to participate}

This study was approved by the Ethics Committee of Shengli Oilfield Central Hospital (Dongying, China). Signed informed consent was obtained from the patients or their guardians.

\section{Patient consent for publication}

Not applicable.

\section{Competing interests}

The authors declare that they have no competing interests.

\section{References}

1. Hirsch FR, Scagliotti GV, Mulshine JL, Kwon R, Curran WJ Jr, Wu YL and Paz-Ares L: Lung cancer: Current therapies and new targeted treatments. Lancet 389: 299-311, 2017.

2. Gridelli C, Rossi A, Carbone DP, Guarize J, Karachaliou N, Mok T, Petrella F, Spaggiari L and Rosell R: Non-small-cell lung cancer. Nat Rev Dis Primers 1: 15009, 2015.

3. Wei X, Zhang K, Qin H, Zhu J, Qin Q, Yu Y and Wang H: GMDS knockdown impairs cell proliferation and survival in human lung adenocarcinoma. BMC Cancer 18: 600, 2018

4. Ferlay J, Shin HR, Bray F, Forman D, Mathers C and Parkin DM: Estimates of worldwide burden of cancer in 2008: GLOBOCAN 2008. Int J Cancer 127: 2893-2917, 2010.

5. Guo T, Zhao S, Wang P, Xue X, Zhang Y, Yang M, Li N, Li Z, $\mathrm{Xu} \mathrm{L}$, Jiang L, et al: YB-1 regulates tumor growth by promoting MACC1/c-Met pathway in human lung adenocarcinoma. Oncotarget 8: 48110-48125, 2017.

6. Yu H, Zhang C, Liu S, Jiang G, Li S, Zhang L, Kang E, Zhang B and $\mathrm{Xu}$ W: Application value of coaxial biopsy system in needle cutting biopsy for focal ground glass-like density nodule. J Cancer Res Ther 14: 1509-1514, 2018

7. Tao G, Jingying Y, Tan G, Xiaotao D and Min C: A novel CT-guided technique using medical adhesive for localization of small pulmonary ground-glass nodules and mixed ground-glass nodules $(\leq 20 \mathrm{~mm})$ before video-assisted thoracoscopic surgery. Diagn Interv Radiol 24: 209-212, 2018

8. Qiu ZX, Cheng Y, Liu D, Wang WY, Wu X, Wu WL and Li WM Clinical, pathological, and radiological characteristics of solitary ground-glass opacity lung nodules on high-resolution computed tomography. Ther Clin Risk Manag 12: 1445-1453, 2016.

9. Kadota K, Villena-Vargas J, Yoshizawa A, Motoi N, Sima CS Riely GJ, Rusch VW, Adusumilli PS and Travis WD: Prognostic significance of adenocarcinoma in situ, minimally invasive adenocarcinoma, and nonmucinous lepidic predominant invasive adenocarcinoma of the lung in patients with stage I disease. Am J Surg Pathol 38: 448-460, 2014

10. Zhang Y, Tang J, Xu J, Cheng J and Wu H: Analysis of pulmonary pure ground-glass nodule in enhanced dual energy CT imaging for predicting invasive adenocarcinoma: comparing with conventional thin-section CT imaging. J Thorac Dis 9: 4967-4978, 2017.

11. Hiramatsu M, Inagaki T, Inagaki T, Matsui Y, Satoh Y, Okumura S, Ishikawa Y, Miyaoka E and Nakagawa K: Pulmonary ground-glass opacity (GGO) lesions-large size and a history of lung cancer are risk factors for growth. J Thorac Oncol 3 : 1245-1250, 2008

12. Chae HD, Park CM, Park SJ, Lee SM, Kim KG and Goo JM: Computerized texture analysis of persistent part-solid ground-glass nodules: Differentiation of preinvasive lesions from invasive pulmonary adenocarcinomas. Radiology 273: 285-293, 2014.

13. Liang J, Xu XQ, Xu H, Yuan M, Zhang W, Shi ZF and Yu TF: Using the CT features to differentiate invasive pulmonary adenocarcinoma from pre-invasive lesion appearing as pure or mixed ground-glass nodules. Br J Radiol 88: 20140811, 2015.
14. Son JY, Lee HY, Lee KS, Kim JH, Han J, Jeong JY, Kwon OJ and Shim YM: Quantitative CT analysis of pulmonary ground-glass opacity nodules for the distinction of invasive adenocarcinoma from pre-invasive or minimally invasive adenocarcinoma. PLoS One 9: e104066, 2014

15. Oda S, Awai K, Murao K, Ozawa A, Yanaga Y, Kawanaka K and Yamashita Y: Computer-aided volumetry of pulmonary nodules exhibiting ground-glass opacity at MDCT. AJR Am J Roentgenol 194: 398-406, 2010.

16. Yoshizawa A, Motoi N, Riely GJ, Sima CS, Gerald WL, Kris MG, Park BJ, Rusch VW and Travis WD: Impact of proposed IASLC/ ATS/ERS classification of lung adenocarcinoma: Prognostic subgroups and implications for further revision of staging based on analysis of 514 stage I cases. Modern pathology 24: 653-664, 2011.

17. Russell PA, Wainer Z, Wright GM, Daniels M, Conron M and Williams RA: Does lung adenocarcinoma subtype predict patient survival? A clinicopathologic study based on the new International Association for the Study of Lung Cancer/American Thoracic Society/European Respiratory Society international multidisciplinary lung adenocarcinoma classification. J Thorac Oncol 6: 1496-1504, 2011.

18. Zhang Y, Shen Y, Qiang JW, Ye JD, Zhang J and Zhao RY: HRCT features distinguishing pre-invasive from invasive pulmonary adenocarcinomas appearing as ground-glass nodules. Eur Radiol 26: 2921-2928, 2016.

19. Gao F, Li M, Sun Y, Xiao L and Hua Y: Diagnostic value of contrast-enhanced CT scans in identifying lung adenocarcinomas manifesting as GGNs (ground glass nodules). Medicine (Baltimore) 96: e7742, 2017.

20. Li M, Gao F, Jagadeesan J, Gill RR, Hua Y and Zheng X: Incremental value of contrast enhanced computed tomography on diagnostic accuracy in evaluation of small pulmonary ground glass nodules. J Thorac Dis 7: 1606-1615, 2015.

21. Travis WD, Brambilla E, Noguchi M, Nicholson AG, Geisinger KR, Yatabe Y, Beer DG, Powell CA, Riely GJ, Van Schil PE, et al: International association for the study of lung cancer/American thoracic society/European respiratory society international multidisciplinary classification of lung adenocarcinoma. J Thorac Oncol 6: 244-285, 2011.

22. Perandini S, Soardi GA, Motton M, Augelli R, Dallaserra C Puntel G, Rossi A, Sala G, Signorini M, Spezia L, et al: Enhanced characterization of solid solitary pulmonary nodules with Bayesian analysis-based computer-aided diagnosis. World J Radiol 8: 729-734, 2016.

23. She Y, Zhao L, Dai C, Ren Y, Zha J, Xie H, Jiang S, Shi J, Shi S, Shi W, et al: Preoperative nomogram for identifying invasive pulmonary adenocarcinoma in patients with pure ground-glass nodule: A multi-institutional study. Oncotarget 8: 17229-17238, 2017.

24. Zhou J, Li Y, Zhang Y, Liu G, Tan H, Hu Y, Xiao J and Shi H: Solitary ground-glass opacity nodules of stage IA pulmonary adenocarcinoma: Combination of 18F-FDG PET/CT and high-resolution computed tomography features to predict invasive adenocarcinoma. Oncotarget 8: 23312-23321, 2017.

25. Lim HJ, Ahn S, Lee KS, Han J, Shim YM, Woo S, Kim JH, Yie M, Lee HY and YiCA: Persistent pure ground-glass opacity lung nodules $\geq 10 \mathrm{~mm}$ in diameter at CT scan: Histopathologic comparisons and prognostic implications. Chest 144: 1291-1299, 2013.

26. Goo JM, Park CM and Lee HJ: Ground-glass nodules on chest $\mathrm{CT}$ as imaging biomarkers in the management of lung adenocarcinoma. AJR Am J Roentgenol 196: 533-543, 2011

27. Zhang T, Pu XH, Yuan M, Zhong Y, Li H, Wu JF and Yu TF Histogram analysis combined with morphological characteristics to discriminate adenocarcinoma in situ or minimally invasive adenocarcinoma from invasive adenocarcinoma appearing as pure ground-glass nodule. Eur J Radiol 113: 238-244, 2019.

28. Yoon HE, Fukuhara K, Michiura T, Takada M, Imakita M, Nonaka K and Iwase K: Pulmonary nodules $10 \mathrm{~mm}$ or less in diameter with ground-glass opacity component detected by high-resolution computed tomography have a high possibility of malignancy. Jpn J Thorac Cardiovasc Surg 53: 22-28, 2005.

29. Lee SM, Park CM, Goo JM, Lee HJ, Wi JY and Kang CH: Invasive pulmonary adenocarcinomas versus preinvasive lesions appearing as ground-glass nodules: differentiation by using CT features. Radiology 268: 265-273, 2013.

30. Hu L, Pan Y, Zhou Z and Gao J: Application of positron emission tomography-computed tomography in the diagnosis of pulmonary ground-glass nodules. Exp Ther Med 14: 5109-5113, 2017.

This work is licensed under a Creative Commons Attribution-NonCommercial-NoDerivatives 4.0 International (CC BY-NC-ND 4.0) License. 